\title{
SUSCEPTIBILIDAD DE MOVIMIENTOS EN MASA EN EL VALLE DE SOCOROMA, PRECORDILLERA ANDINA DE ARICA Y PARINACOTA*
}

\author{
MASS MOVEMENT SUSCEPTIBILITY IN THE SOCOROMA VALLEY, \\ ANDEAN PRECORDILLERA OF ARICA AND PARINACOTA
}

\author{
Alan Rodríguez, ${ }^{* *}$ Alejandro Tapia ${ }^{* * *}$ y Cristián Albornoz, ${ }^{* * * *}$
}

\begin{abstract}
Se presenta un análisis de susceptibilidad de movimientos en masa en el sector del valle-quebrada de Socoroma, región de Arica y Parinacota (Chile). Este trabajo analiza factores condicionantes de la inestabilidad del terreno (litología, pendientes, sentido de las pendientes, lineamientos estructurales, distancia a los drenajes principales, altura, inventario de movimientos en masa e Índice Normalizado de Diferencias Vegetacionales). El resultado es la sectorización de la susceptibilidad de movimientos en masa en el área estudiada, según el Índice de Susceptibilidad obtenido a partir del método de Evaluación Multicriterio de las Jerarquías Analíticas. Sobre la base de los resultados obtenidos respecto del fenómeno de movimiento de masas, se establece que el pueblo de Socoroma se emplaza en un sector de baja susceptibilidad, a diferencia del sector sureste del área considerada que concentra los mayores grados de susceptibilidad al fenómeno estudiado, sección en que se encuentra un segmento de la carretera internacional 11-CH, la principal vía de comunicación de Bolivia con el litoral del océano Pacífico, y la más importante conexión entre los poblados altoandinos de la provincia de Parinacota con los valles bajos y la ciudad de Arica.
\end{abstract}

Palabras clave: Susceptibilidad, movimientos en masa, valle de Socoroma.

This article analyzes the susceptibity for mass movement in the Socoroma Valley, Arica and Parinacota region (Chile). This paper addreses the determinants of ground instability (lithology, slope, slope directions, structural lineaments, main drains distance, height, landslide inventory and Normalized Difference Vegetation Index). The result is the sectoring of susceptibility for landslides in the valley, according to the susceptibility index, obtained from the method Multi-criteria Evaluation with Analytical Hierarchy Process. Based on the results the mass movement, it was established that the Socoroma village is placed in an area of low susceptibility, while the south east sector of the considered area presents greater degrees of susceptibility; where the 11-CH international highway passes, the principal link between Bolivia and the coast of the Pacific Ocean, and the most important road connection between the Andean towns of the province of Parinacota with the lower valleys and the city of Arica.

Key words: Susceptibility, mass movement, Socoroma valley.

\section{Introducción}

La precordillera andina del extremo norte de Chile está expuesta a fenómenos naturales como sismos y lluvias intensas, presentándose estas últimas fundamentalmente en temporada estival (Aceituno, 1996). Estos fenómenos pueden producir movimientos en masa, que se encuentran mayoritariamente asociados a la susceptibilidad del terreno a ser afectado por desplazamientos frente a estos eventos desencadenantes.

Los fenómenos naturales de movimientos de masa a lo largo de la cordillera de los Andes, según Suárez (2010), dependen de las condiciones geomorfológicas, geológicas y del clima imperante en los diversos sectores de esta macroforma. Este autor menciona cuatro elementos como los más importantes para la ocurrencia de los movimientos en masa: el relieve, la sismicidad, la meteorización y las lluvias intensas. A su vez, Hauser (1993) considera que es fundamental conocer los factores climáticos, geomorfológicos, geológicos y tectónicos que tienen lugar en los sectores de montaña, y señala que los ambientes andinos, constituidos por secuencias sedimentarias y volcanoclásticas sometidas a intensas deformaciones tectónicas poscretácicas, junto a terrenos vinculados a actividad eruptiva terciaria a moderna, resultan especialmente favorables para el desarrollo de procesos de remoción en masa. De acuerdo con lo expuesto por ambos autores se entiende

\footnotetext{
* Agradecimientos Convenio de Desempeño UTA-MINEDUC.

** Investigador independiente. Correo electrónico: alanrodriguezvaldivia@ gmail.com

*** Universidad de Tarapacá, Departamento Ciencias Históricas y Geográficas, Arica, Chile. Correo electrónico: atapia@uta.cl

***** Heidelberg Center para América Latina, Santiago, Chile. Correo electrónico: albornoz.espinoza@ gmail.com
} 
que los movimientos en masa están influidos por distintos factores, o elementos condicionantes, que posibilitan la ocurrencia de los fenómenos. Entre los factores señalados por Suárez (2010) y Hauser (1993) se pueden diferenciar claramente los de tipo interno (sismicidad, litología y estructura geológica) y externo (gravedad y clima imperante).

Diversos autores (Kawabata y Bandibas, 2009; Ram Avtar et al., 2013; Intarawichian y Dasananda, 2010; Aflidason et al., 2005; Díaz et al., 2012; Gil y Campo, 2012; Royán y Vilaplana, 2012) concuerdan en considerar una serie de factores para el análisis de movimientos en masa realizados mediante la aplicación de Sistemas de Información Geográfica (SIG), entre los que se encuentran: pendientes, litología y estructura geológica (principalmente la existencia de fallas). En el presente trabajo se consideran factores como: (1) pendiente, (2) litología, (3) distancia a lineamientos estructurales (fallas), (4) altura, (5) orientación de las pendientes, (6) distancia al drenaje principal, (7) inventario de movimientos en masa y (8) vegetación.

El presente estudio tiene por objetivo sectorizar, a partir de un índice de susceptibilidad, la intensidad de los movimientos en masa en un área circundante al pueblo de Socoroma; busca además contribuir -desde una perspectiva geográfica- al conocimiento y comportamiento espacial de estos fenómenos en la precordillera andina de la región de Arica y Parinacota, para ello se realiza un análisis multicriterio basado en factores tenidos en cuenta en trabajos análogos, así como aquellos considerados relevantes para el área estudiada.

\section{Descripción del valle de Socoroma}

El valle-quebrada de Socoroma (en aymara chukuruma, "agua que corre") se encuentra a una altitud aproximada de $3.070 \mathrm{msnm}$ en la comuna de Putre, unidad administrativa correspondiente a la región de Arica y Parinacota. El área se caracteriza por presentar una serie de estribaciones desprendidas del cordón occidental de la cordillera de los Andes (Seyfried et al., 1998). Socoroma limita por el norte con el río Aroma y el curso alto del río Lluta, por el este con el cerro Pusiri y cerro Peña Grande, quebrada Chalcane y la ruta internacional 11-CH. Hacia el sur con la quebrada Vizcachane, cerro la Cruz y la pampa Tantalcollo; mientras que al oeste con la quebrada Marcuma y cerro Chatiza (Figura 1).

El trabajo se enmarca en un espacio precordillerano andino del extremo norte de Chile que se presenta notablemente accidentado, producto de la acción de las fuerzas tectónicas imperantes en la región (Seyfried et al., 1998; Lavenu, 2006; Stern, 2004). Trabajos anteriores han tratado sobre algunos de los accidentes del terreno en el sector, que han sido abordados mediante mitos locales (Álvarez, 1987) y explicados por la ocurrencia de importantes deslizamientos rotacionales (Montecinos, 1962).

Las condiciones climáticas en este sector corresponden a las propias de un desierto marginal de altura con tendencia tropical (clima BWh según el sistema de clasificación climática de Köppen), influido por lluvias estivales originadas a partir de la acción ejercidas por el "monzón sudamericano" y por el fenómeno de convección térmica que sufren las masas de aire en la estación de verano (Fuenzalida, 1965). El área presenta una precipitación total anual aproximada de $195 \mathrm{~mm}$, concentrándose la mayor parte de esta entre diciembre y marzo, los valores térmicos medios mensuales, que a lo largo del año fluctúan entre $6^{\circ}$ y $9^{\circ} \mathrm{C}$, se presentan influidos por la altitud a que se encuentra el área de estudio, sin embargo si se consideran los valores absolutos, las oscilaciones diarias son muy marcadas, alcanzando valores inferiores a $0{ }^{\circ} \mathrm{C}$ en la noche y superiores a $20^{\circ} \mathrm{C}$ en el día. Por tratarse de un sector árido, su baja humedad relativa durante el día es muy característica, especialmente durante los meses de invierno.

El área de estudio que cubre una superficie aproximada de 2.875 hectáreas fue delimitada basándose en dos rasgos físicos del relieve: (1) pendiente y (2) altitud, rasgos de los que dependen las características principales que presentan las laderas en el sector. Las laderas se constituyen en los relieves fundamentales en los que ocurren movimientos en masa, favorecidos por el ángulo de pendiente, orientación geográfica respecto de la dirección de los vientos dominantes, y estado de meteorización de las rocas superficiales que las conforman. En las laderas del área de estudio se presentan procesos gravitatorios favorecidos por las diferencias de altitud, superiores a $1.000 \mathrm{~m}$, lo que origina relieves con laderas de grandes ángulos de pendiente y con alta probabilidad de que en ellos se desencadenen movimientos en masa.

La quebrada de Socoroma se ubica en la vertiente oeste del cordón occidental de la cordillera de los Andes Centrales, presenta escurrimiento hacia el litoral Pacífico, al confluir con el río Lluta, el sistema hidrográfico más importante de la XV Región. 


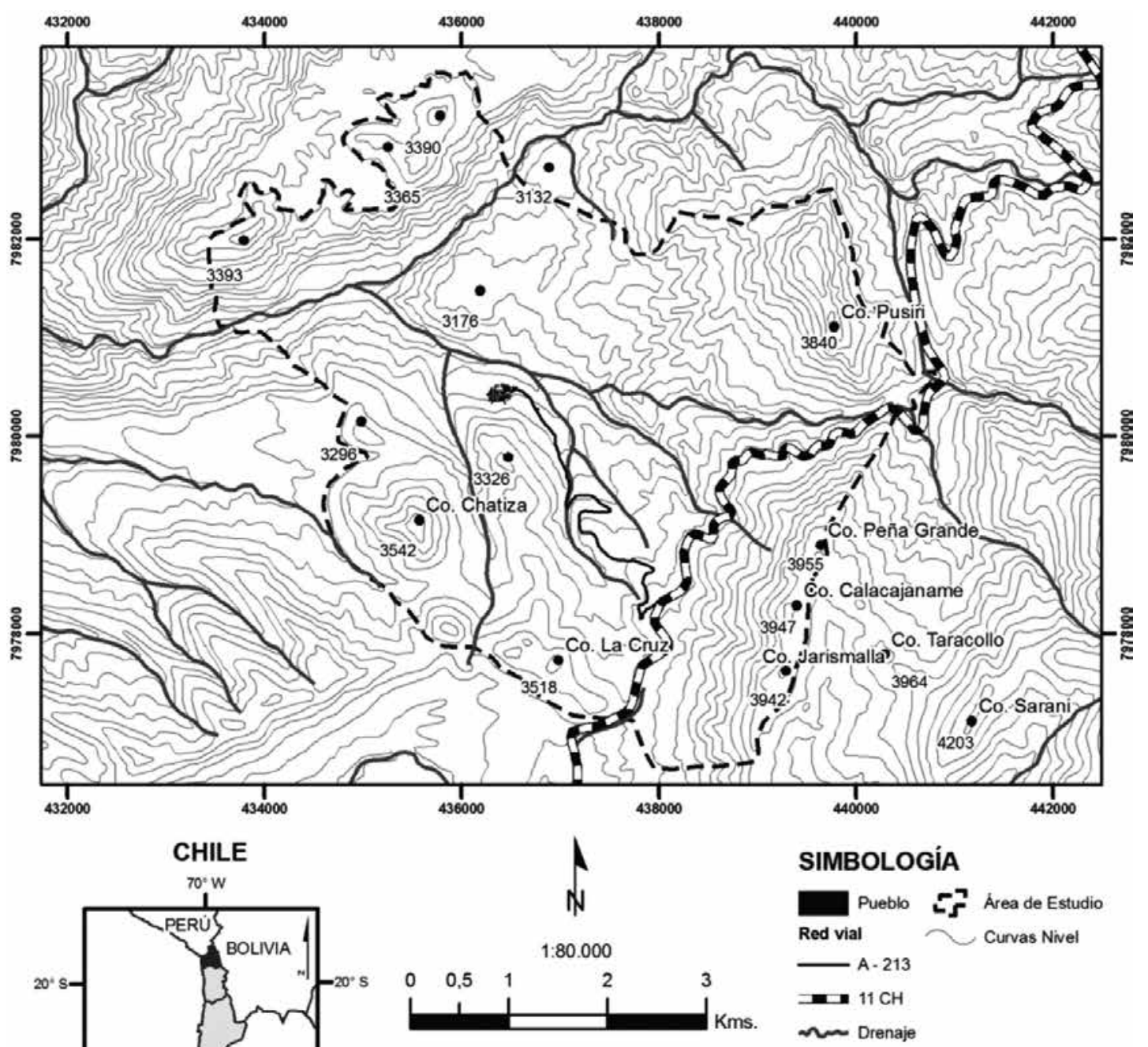

REGIÓN DE ARICA Y PARINACOTA

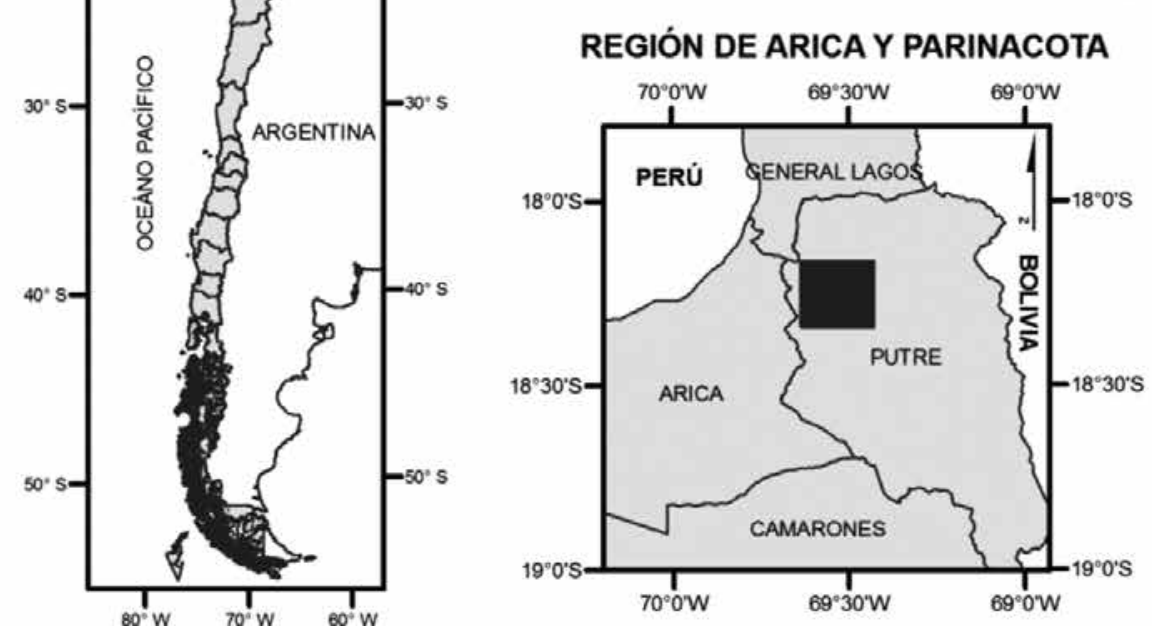

Figura 1. Área de estudio. 
La población que habita Socoroma, como la generalidad de los habitantes de los sectores interiores de la región de Arica y Parinacota, está constituida por población de ascendencia aymara que basa su sustento en el trabajo de la tierra. La práctica de la actividad agrícola llevada a cabo, desde épocas pretéritas, por la población local en un área de montaña tan accidentado, como es la sierra, le ha permitido obtener el mayor provecho de ese espacio al modificar el terreno, creando sistemas de andenerías o pata-pata en sectores de ladera, favoreciendo con ello la expansión de las áreas de cultivos.

El espacio correspondiente al área de estudio posee una vegetación de tipo xerófita, condicionada por el tipo de clima que impera en la precordillera andina, el clima desértico marginal de altura, ante cuyas condiciones la vegetación del sector debe presentar una adaptabilidad extrema. La vegetación autóctona del área se clasifica como propia de los pisos supratropical y orotropical semiáridos según Luebert y Gallardo (2005). Esta vegetación corresponde a matorrales desérticos bajos, matorral xeromórfico con suculentas, matorral denso en especies, matorral subdesértico, y cactáceas. La vegetación alóctona que puede reconocerse en los alrededores del poblado de Socoroma corresponde a eucaliptus, álamos y cipreses.

\section{Materiales y métodos}

Los materiales utilizados en este estudio corresponden a información geográfica digital (datos vectoriales y raster), Sistemas de Información Geográfica (SIG), cartas topográficas e instrumentos de campo. Para lograr la información geográfica se utilizó una imagen satelital LANDSAT 7 ETM del norte de Chile (tamaño de pixeles de $30 \mathrm{x}$ $30 \mathrm{~m}$ ) correspondiente al 2010, a partir de la cual se obtuvo el Índice Normalizado de Diferencias Vegetales (NDVI); imágenes Google Earth georreferenciadas en un SIG, con estas se digitalizaron archivos vectoriales, un Modelo de Elevación Digital (MDE) ASTERGDEM (tamaño de pixeles 30x30 m de superficie terrestre) del 2009, para modelar pendientes, altura, orientación de las pendientes y crear un mapa topográfico del área de estudio. También se utilizó información topográfica base del lugar estudiado, obtenida a partir de las cartas topográficas del Instituto Geográfico Militar (IGM) escala 1:50.000. Los instrumentos de campo, a su vez, permitieron verificar y levantar datos en la localidad y sectores aledaños. El conjunto de información obtenida por las distintas fuentes señaladas se trabajó mediante un SIG, con la finalidad de obtener el índice de susceptibilidad mediante la aplicación del análisis multicriterio.

Para establecer una jerarquía y pesos a cada factor condicionante se utilizó el Método de Evaluación Multicriterio de las Jerarquías Analíticas (Saaty, 1980; Hervás y Barredo, 2001). Primero, a base de una matriz cuadrada, se estableció el peso para cada clase de cada factor. Seguidamente se confeccionó una matriz cuadrada donde se estableció de manera subjetiva el peso de cada factor. Después, para establecer la razón numérica del rango de susceptibilidad se utilizó la ecuación:

$$
L S I=\sum_{i=1}^{n}(W i \times R i)
$$

Donde:

LSI = Índice de Susceptibilidad a los Deslizamientos (originalmente landslide susceptibility index).

$\mathrm{W} i=$ Peso del factor.

$\mathrm{R} i=$ Peso de la clase.

Una vez obtenido el índice se procedió en un SIG a utilizar el método de análisis multicriterio. Este permitió ingresar los resultados (pesos) obtenidos de análisis jerárquico a cada capa raster, correspondiente a cada factor manipulado. Para lograrlo, los archivos vectoriales, por ejemplo el inventario de movimientos en masa, fueron convertidos a archivos raster con tamaño de pixel de $900 \mathrm{~m}^{2}$, tamaño utilizado en todos los archivos incorporados en el estudio. Luego, la aplicación procedió a calcular, automáticamente, la suma de los pixeles de las ocho capas raster. $\mathrm{La}$ aplicación procedió a integrar toda esta información en una sola capa raster, correspondiente al índice de susceptibilidad obtenido con anterioridad.

Finalmente, para alcanzar una representación y visualización óptima de la información se procedió a clasificar el índice en un SIG, a partir de límites naturales o natural breakes, en cinco rangos de susceptibilidad:

- 0,067-0,132 (Muy bajo)

- 0,132-0,174 (Bajo)

- 0,174-0,231 (Moderado)

- 0,231-0,317 (Alto)

- 0,317-0,470 (Muy alto)

Toda la información obtenida fue representada en una cartografía de salida 1:50.000. 


\section{Descripción de los factores}

\section{Litología}

El valle de Socoroma está compuesto principalmente por rocas de tipo volcánico, andesitas e ignimbritas cubren el 80,7\% del área estudiada, mientras que depósitos de coluvión y remoción de masas cubren el $14 \%$, como se visualiza en la Figura 2. Las rocas de tipo volcánico poseen una alta susceptibilidad a ser afectadas por procesos de desestabilización que desencadenan movimientos en masa, más aún, los depósitos de remoción en masa y coluviones son los que presentan la mayor probabilidad a ser removilizados o muestran inestabilidad (Suárez, 2010). En el área se pueden distinguir, según García et al. (2004), siete tipos de rocas:

Las rocas andesitas corresponden a las unidades geológicas de las formaciones Oxaya (andesita Socoroma), Lupica, y volcanes y remanentes de volcanes del Mioceno inferior. La andesita Socoroma corresponde a un estrato gris pardo de $120 \mathrm{~m}$ de espesor, expuesto en el sector norte y oeste de Socoroma. Las andesitas de la formación Lupica corresponde a lavas propilitizadas y oxidadashematitizadas que afloran al Este de Socoroma. El tercer tipo de andesita corresponde a un conjunto de rocas pertenecientes a edificios volcánicos, mal a medianamente conservados y remanentes de estratovolcanes.
Las ignimbritas que cubren el área estudiada corresponden a unidades geológicas de las formaciones Lauca, Oxaya y Lupica. La primera corresponde a un depósito de flujo piroclástico de colores rosado, gris y pardo rojizo de gran extensión areal en la alta cordillera, precordillera y depresión central. La segunda corresponde a mantos de tobas ignimbríticas de depósitos de flujo piroclásticos. La tercera está constituida por una sucesión de tobas, predominantemente vítreas y de cristales, que alcanza $1.000 \mathrm{~m}$ de espesor, que está alterada hidrotermalmente y no presenta intercalación sedimentaria, lo que sugiere un solo estrato ignimbrítico potente.

Las rocas sedimentarias de arenisca y grava y el intrusivo de diorita corresponden a las unidades geológicas de las formaciones Lupica, Huayllas e Intrusivos del Mioceno medio, respectivamente. $\mathrm{La}$ primera unidad corresponde a sucesiones formadas por 400 a $600 \mathrm{~m}$ de areniscas, fangolitas y calizas, de colores verde, gris, pardo y amarillo con intercalaciones de conglomerado. La segunda pertenece al miembro inferior de la secuencia Copaquilla, que está formada por hasta $20 \mathrm{~m}$ de espesor de arenas, limos y gravas clastosoportadas, semiconsolidados, de colores pardo-rojizo, gris y gris-verdoso. La tercera está conformada por conjuntos de cuerpos plutónicos e hipabisales, de extensión restringida, con formas subcirculares.

Se ha agrupado un conjunto de rocas que presentan la mayor probabilidad de inestabilidad,

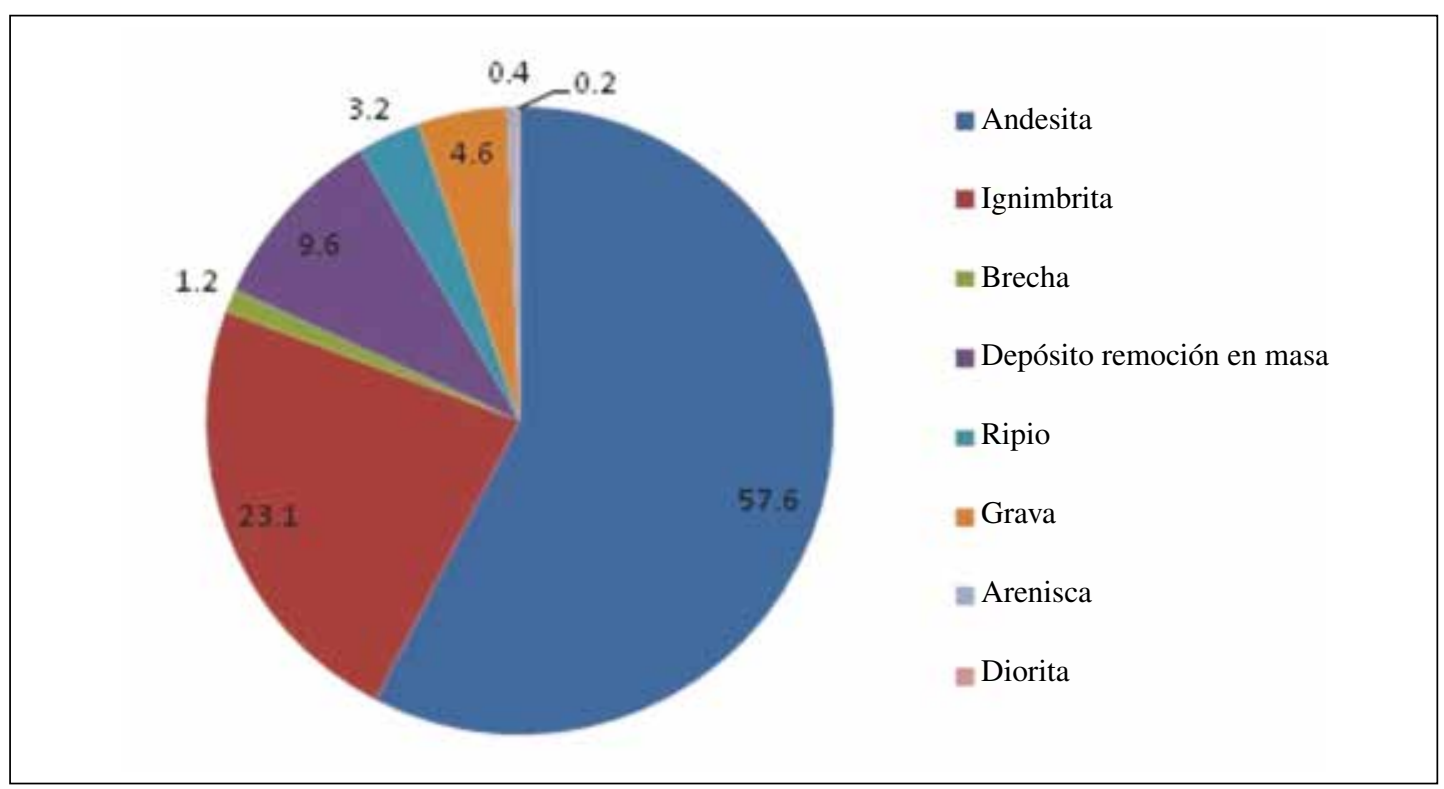

Figura 2. Rocas que cubren el área estudiada, expresadas en porcentaje. Elaboración basada en García et al. (2004). 
conformado por brechas, depósitos de remoción de masas y ripio correspondientes a las unidades geológicas de depósitos coluviales, depósitos de remoción en masa del Plioceno-Cuaternario y depósitos aluviales del Pleistoceno. El primer grupo corresponde a depósitos caóticos, no consolidados, principalmente monomícticos y oligomícticos, de origen gravitatorio, y constituidos por bloques, bolones, rodados, arenas y limos. El segundo corresponde a depósitos de deslizamiento y avalanchas gravitacionales, de variadas dimensiones, con superficies de hasta $30 \mathrm{~km}^{2}$, expuestos principalmente en bordes de ladera y escarpes abruptos en quebradas profundas. El tercer grupo compuesto por grava, brechas matriz soportadas, semiconsolidadas, con intercalaciones de arena, limo y arcilla. Los clastos de origen predominantemente volcánico, y los depósitos se asocian a acumulaciones como productos de flujos de barro y/o detritos, gatillados por precipitaciones torrenciales.

\section{Lineamientos estructurales}

En el área estudiada se reconocen tres fallas de tipo inverso, distinguiéndose la falla inversa denominada Socoroma. Esta falla se debe a la deformación genérica del Mioceno-Holoceno en la alta cordillera y se localiza al Este del poblado de Socoroma. La falla monta hacia el Oeste la Formación Lupica, sobre la Formación Oxaya. Es posterior a 18 Ma., y su actividad temprana y occidental está sellada por lavas del volcán Quevilque, mientras que su actividad tardía está sellada por lavas del Mioceno Medio (García et al., 2004).

Los rasgos geomorfológicos de la falla Socoroma (Figura 3) infieren fallamiento de tipo inverso, cuyos estratos mantean hacia el Este, hacia donde se encuentran pliegues y fallas menores. Se puede inferir que correspondería a un sobrescurrimiento de una falla contraccional con dirección Oeste, cuyo plano de falla presenta facetas triangulares producto de la erosión.

\section{Geomorfodinámica}

La precordillera, donde se localiza el valle de Socoroma, se caracteriza por conformar una meseta alargada que varía entre 20 a $35 \mathrm{~km}$ de ancho, que en altitud está disectada por quebradas profundas (Lluta, Azapa y Camarones). En el sector norte, en la sierra de Huaylillas y pampa de Oxaya, producto de las fuerzas compresivas generadas por la subducción, existe una superficie suavemente plegada. En tanto al Sur, en la pampa de Sucuna, un monoclinal suave mantea $2^{\circ}$ a $3^{\circ}$ al Oeste (García et al., 2004).

La erosión de tipo fluvial es característica en aquellos sectores elevados, evidenciándose por la red de cárcavas. Cuando las precipitaciones aumentan

NE

SW

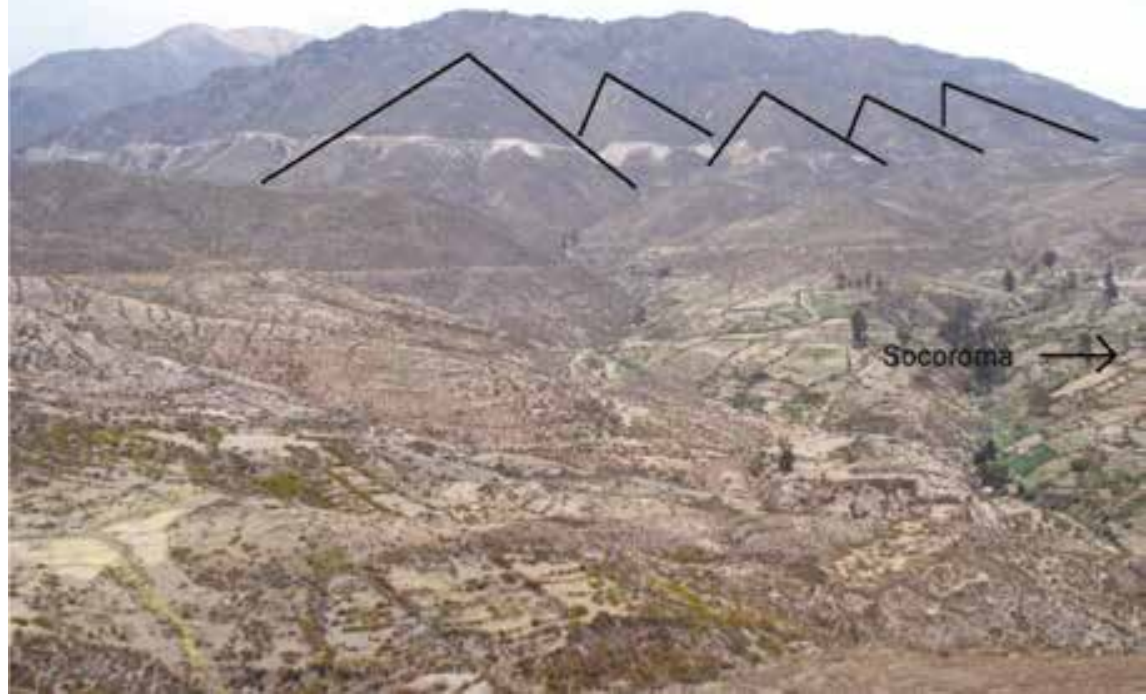

Figura 3. Morfología de facetas triangulares en falla Socoroma, plano de falla. 
en temporada estival, las aguas-lluvia se canalizan en la conformación antes mencionada y comienzan los procesos de remoción del sedimento fino y grueso que se encuentra en estos lugares, siendo transportados y depositados en los sectores bajos. Producto de la remoción del material, los flujos de detritos crean formas características de lóbulos en los pies de la ladera.

La erosión fluvial posee estrecha relación con la cantidad de precipitación caída en la alta cordillera y sectores elevados, producto de ello el caudal de los ríos presenta variaciones, provocándose una mayor o menor erosión de acuerdo con los montos de los escurrimientos superficiales. Los principales escurrimientos superficiales permanentes de agua que se presentan en el área de estudio son el río Aroma y el río Socoroma, los que al aumentar sus caudales, cuando las precipitaciones son muy intensas, erosionan las paredes que conforman sus laderas e inundan sectores más elevados del cauce. La disposición de los materiales que componen el lecho de los ríos es de tipo caótico, constituidos por rodados, grava, arena y en menor cantidad limo y arcilla. La disposición heterogénea de los clastos demuestra que los flujos de agua pueden evolucionar a verdaderos torrentes, cuando el caudal aumenta considerablemente en temporada estival.

La erosión eólica afecta sobre todo a aquellos lugares desprovistos de cubierta vegetal y donde el material sedimentario fino es abundante. Las laderas expuestas hacia los sectores noroeste y oeste presentan una erosión más intensa, debido a la dirección predominante de los vientos locales en el valle.

En síntesis, la morfodinámica en el área estudiada se debe a una asociación de procesos fluviales, pluviales, eólicos y de meteorización que modelan la fisiografía actual del valle y sus alrededores.

\section{Geomorfometría}

Los parámetros morfométricos analizados fueron seleccionados por la capacidad que poseen para incidir en los procesos de inestabilidad de laderas, como es el caso de los ángulos de pendiente, altitud y sentido de las pendientes.

Las pendientes son un factor que probablemente poseen un alto grado de influencia en los procesos gravitatorios, producto de que laderas o taludes con altos ángulos de pendientes presentan mayor probabilidad a la desestabilización, sin embargo se deben considerar además los procesos de meteorización, fracturamiento, evento detonante entre otros más que favorecen la movilización del material. Las pendientes fueron agrupadas según la clasificación establecida por Suárez (2010: Tabla 13.3), que considera los siguientes valores: Muy Baja $\left(0^{\circ}-8,5^{\circ}\right)$, Baja $\left(8,5^{\circ}-16,7^{\circ}\right)$, Mediana $\left(16,7^{\circ}\right.$ $\left.26,6^{\circ}\right)$, Alta $\left(26,6^{\circ}-45^{\circ}\right)$ y Muy Alta $\left(>45^{\circ}\right)$.

La altitud se consideró teniendo presente las diferencias de elevación que se observaron en el trabajo de campo. Mediante un Modelo Digital de

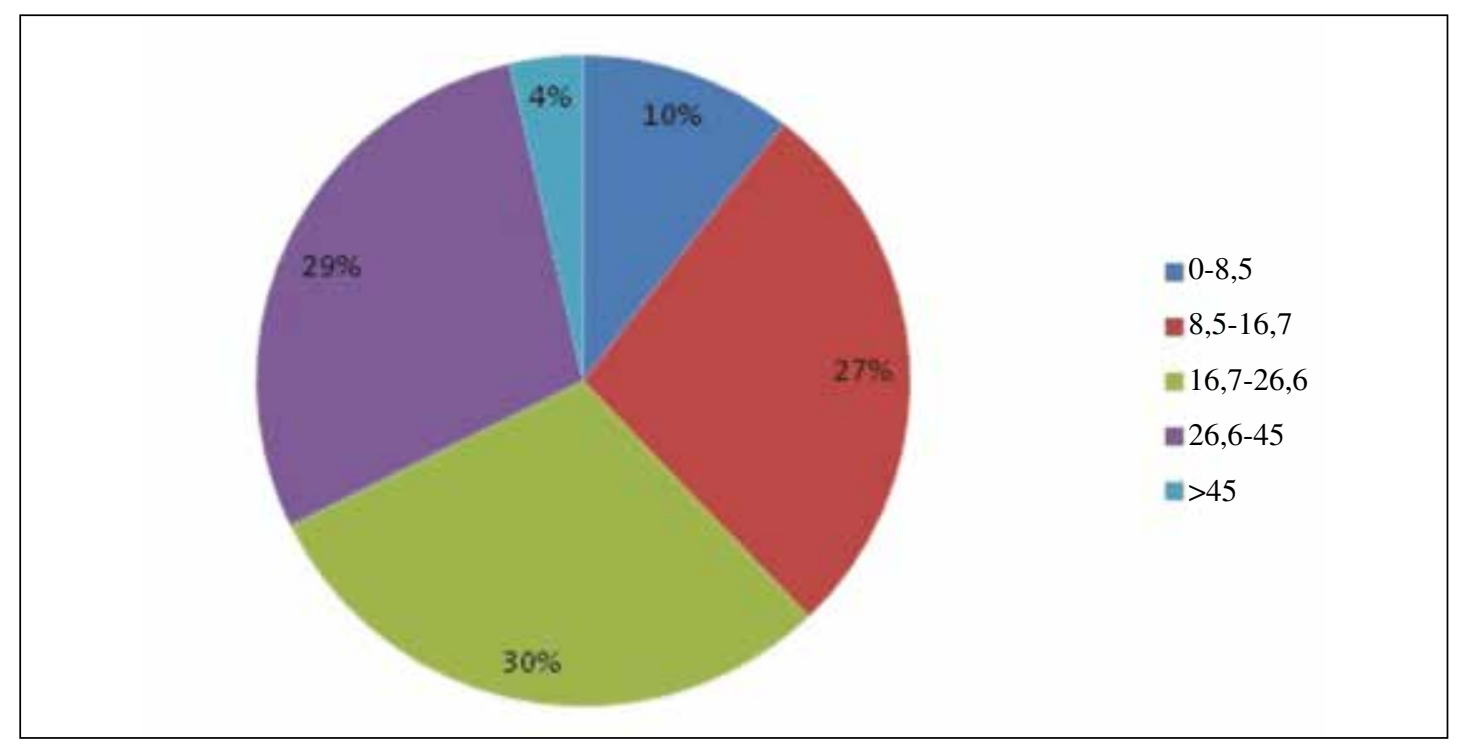

Figura 4. Superficie que cubre cada rango de pendiente en el área de estudio. 
Elevación (MDE) se logró identificar los sectores con mayor elevación en el área estudiada, los que se localizan en el sector este, donde se encuentran la falla Socoroma y parte del trazado de la carretera internacional 11-CH.

El sentido de las pendientes se consideró para la determinación de la erosión de tipo eólico, producida por los vientos locales de valle con sentido principalmente oeste. Si bien los procesos erosivos de tipo eólico no son tan considerables, pueden tener una incidencia en la movilización o removilización de pequeñas partículas, como arena o limo, que desgastan la roca por acción abrasiva.

La erosión lineal se centra principalmente en el talweg de los dos escurrimientos superficiales presentes en el área de estudio, el río Socoroma y el río Aroma, el primero escurre con sentido sureste-noroeste y el segundo en sentido este-oeste. También se presentan algunas corrientes superficiales efímeras que se localizan principalmente en el sector sur-sureste del área estudiada, en el que el agua en periodo de lluvias intensas erosiona, remueve y transporta material residual a sectores bajos.

\section{Inventario de movimientos en masa}

En el valle de Socoroma se distinguen tres tipos de movimientos en masa: caídos de roca y/o derrubios, vuelcos y flujos de detritos. Los dos primeros ocurren debido a fenómenos de orden gravitatorio relacionados principalmente con los procesos de meteorización. El tercero es producto de la movilización y removilización de material detrítico. Es preciso considerar que para los tres tipos de movimiento en masa la gravedad participa directa o indirectamente, por lo que los flujos detríticos no presentan un solo agente actuante, sino que participan complementariamente agua y gravedad (De Pedraza et al., 1996).

Los caídos de roca afectan en gran parte aquellos sectores denudados o parcialmente desprovistos de vegetación, con relieves de altas pendientes y con marcadas diferencias de altura. El sector noroeste posee estas características, de manera que la ladera presenta cornisa, talud y enlace basal, lo que confirma el hecho de que este sector posea vertientes activas. El sector sureste que posee características parecidas se comporta como una vertiente activa, de allí que en su base exista una gran cantidad de testigos de roca que descendieron por la ladera, producto de la gravedad y/o transportados por el agua (obsérvese Figura 5). En la ladera oriental (vista desde la carretera internacional 11-CH hacia el pueblo de Socoroma) las características de la pendiente son parecidas, pero es un lugar con mayor susceptibilidad de ocurrencia de movimientos, ya que posee depósitos de tipo coluvión en la base, que son muy propensos a la formación de nuevos deslizamientos (Suárez, 2010).

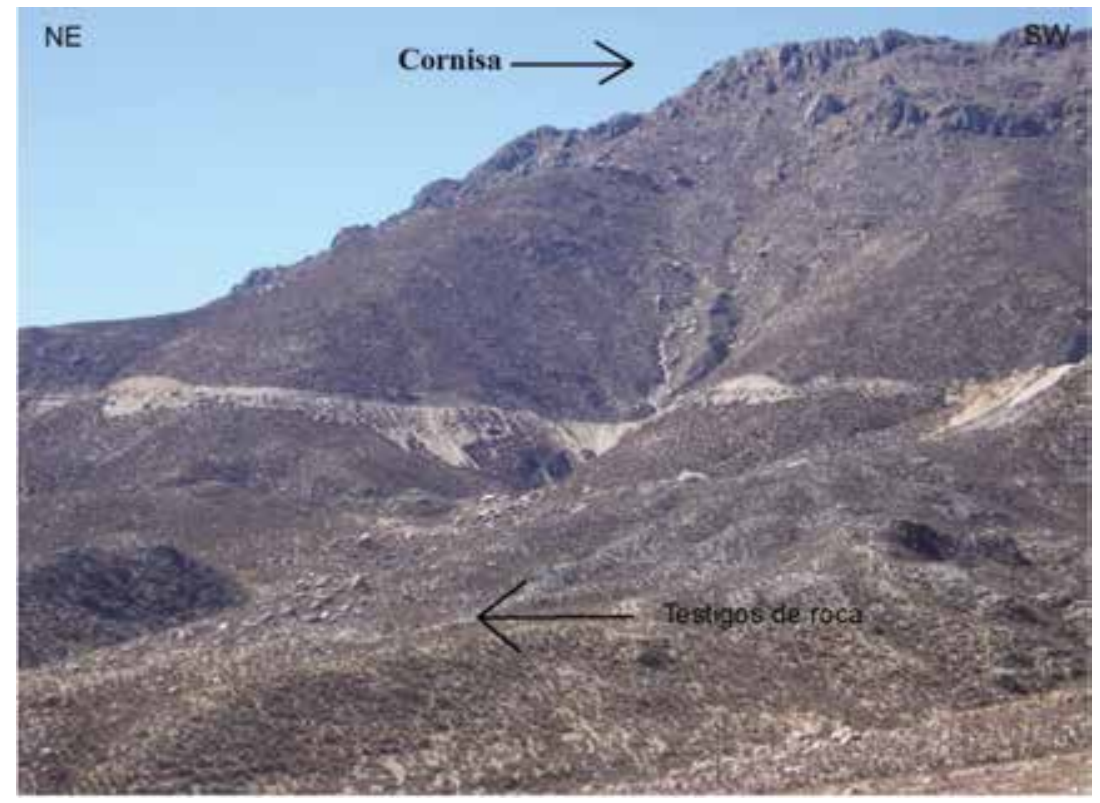

Figura 5. Caída de bloques y flujos de detritos. 
Los vuelcos son característicos de los sectores noroeste, sureste y este, donde los afloramientos rocosos sufren una meteorización intensa, condición que favorece la formación de diaclasas verticales, creando planos propicios para el vuelco en las estructuras más expuestas del saliente rocoso (obsérvese Figura 6).

Los flujos de detritos se canalizan en las cárcavas o regueros de laderas con notable pendiente y alta meteorización. Principalmente son sectores con afloramiento de roca madre en descomposición, en los que se puede formar una capa de suelo con material de tipo grava y arena gruesa. Los sectores noroeste y sureste del área de estudio poseen condiciones y depósitos de este tipo.

Las avalanchas de barro o flujos de barro corresponden a un tipo de movimiento que tiende a producirse en los drenajes principales del valle. En el sector noroeste, donde confluyen el río Aroma y el río Socoroma, el agua puede remover y transportar gran cantidad de clastos y sedimentos, los que se agregan al material alóctono del flujo.

No es de extrañar que grandes desastres ocurridos en la zona andina estén relacionados con flujos de lodo, fenómeno que en el Sur del Perú y Norte de Chile se les denomina con distintos términos: huaicos, lloclla y aluviones (Hauser, 1993, 1997; Golubev, 1969).

Para el caso del presente estudio se incorporaron los escurrimientos principales en el archivo raster de distancia a drenajes, para representar la susceptibilidad del proceso descrito anteriormente.

Es importante aclarar que las avalanchas o flujos de barro pueden integrar material de los flujos residuales y de los mismos caídos de roca; por consiguiente, tanto el cauce del río Aroma como cauce el río Socoroma son propensos a presentar flujos de barro.

\section{Índice Normalizado de Diferencias Vegetales (NDVI)}

El Índice Normalizado de Diferencias Vegetales se utilizó para reconocer los suelos según la cubierta vegetal que presentan, de acuerdo con la cobertura se clasificaron en cuatro rangos. El primero corresponde a suelo desnudo, constituido por sectores de salientes rocosos, caminos y área ocupada por el pueblo de Socoroma. El segundo rango comprende aquella superficie parcialmente cubierta por matorrales dispersos. El tercero presenta matorrales y cactáceas con mayor densidad. Y el cuarto muestra las áreas ocupadas por la población local para el cultivo en andenerías.

\section{Superposición de factores a movimientos en masa}

A continuación se presenta la razón numérica, basada en el Análisis Jerárquico de Saaty (Tabla 1),

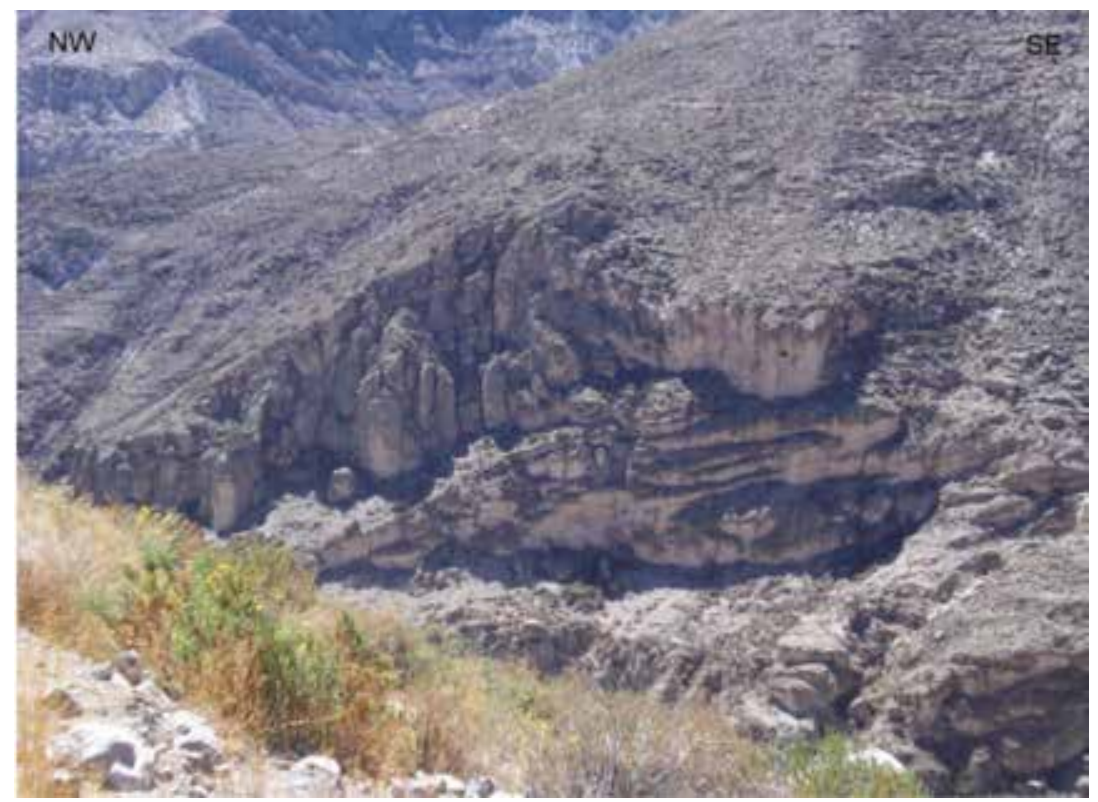

Figura 6. Diaclasas verticales que favorecen los vuelcos. 
para cada factor que condiciona los movimientos en masa y sus correspondientes clases. Los valores numéricos obtenidos están expresados en decimales y con sus respectivos índices de inconsistencia. Esto con la intención de mostrar los resultados con claridad y que permita comprender de mejor manera la información que entrega la Figura 8.

Los datos espacializados del índice de susceptibilidad (Tabla 2), obtenido a partir de los resultados de la Tabla 1, permitieron obtener la superficie de cada grado de susceptibilidad. La clase que presenta la más baja susceptibilidad a movimientos cubre el $43 \%$ de la superficie estudiada. La unión de las dos clases más bajas de susceptibilidad cubre el $70 \%$; mientras que la clase moderada solo representa el $22 \%$ del total. De hecho, la suma del porcentaje de las dos clases más altas no supera el $8 \%$ del total de la superficie considerada.

Por consiguiente, se puede entender que del 100\% del área estudiada solo $30 \%$ corresponde a rangos de susceptibilidad moderado a muy alto. El $22 \%$ de probabilidad moderada está considerablemente relacionada con los espacios de mayor altitud, depósitos de coluvión y/o remoción de masas, y con la localización de la Falla Socoroma. Mientras que el $8 \%$ restante corresponde a los sectores de mayor pendiente de laderas, incluyendo los salientes rocosos proclives a provocar movimientos de tipo caídos de roca.

Es importante considerar, de acuerdo a los resultados obtenidos, que los relieves fallados cumplen un rol fundamental en los procesos de movimientos de ladera. Complementando los trabajos de campo, la información geológica recopilada y los resultados obtenidos del modelo de susceptibilidad, se logró identificar depósitos de coluvión localizados al pie del plano de falla Socoroma, procesos gravitatorios activos en las cárcavas que disectan el plano de falla y salientes rocosas a lo largo de la cornisa que suministran material en proceso de desintegración con el potencial de producir caídos de roca y/o proporcionar fragmentos que podrían incorporarse a flujos residuales.

Al complementar la información de la Tabla 2 con la sectorización de susceptibilidad que muestra la Figura 8, se distingue que los sectores donde se localizan los puntos críticos, que corresponden a los lugares en que el fenómeno de movimiento en masa se manifestó dentro del área estudiada y que fueron catastrados por ONEMI y Carabineros de Chile en 1997, 1999 y 2011, concuerdan con las áreas que presentan mayores rangos de susceptibilidad de ser afectados por movimientos de masas. Esto se puede explicar por dos razones. Primero, porque el espacio donde se localiza la mayor cantidad de puntos críticos es el mismo donde se posiciona la falla Socoroma, puntos que siguen una dirección paralela a dicha falla. Segundo, por la ubicación de la ruta internacional 11- $\mathrm{CH}$, cuya construcción significó una alteración en las condiciones naturales de las pendientes e implicó en muchos casos crear mayor inestabilidad en las vertientes intervenidas, por ello cada temporada estival tanto Carabineros de Chile como la ONEMI tienen la misión de informar los cortes que se producen a lo largo de esta carretera por acción de las aguas lluvia y su posterior escurrimiento.

\section{Discusión}

Los resultados obtenidos demuestran que los espacios correspondientes a la sierra de la región de Arica y Parinacota son susceptibles a la ocurrencia de movimientos en masa, debido a tres razones principales:

Primero, los procesos tectónicos que han configurado el relieve andino generaron relieves asimétricos y con diferencias de altitud considerable en los espacios precordilleranos, posibilitando una morfometría de pendientes abruptas en el área de estudio. Este factor, según Suárez (2010), es uno de los que condiciona la susceptibilidad del terreno a producir movimientos. De acuerdo con los resultados obtenidos en el presente estudio, se puede inferir que las pendientes juegan un rol importante al momento de analizar estos fenómenos, ya que, por ejemplo, los movimientos en masa de tipo flujo de detrito se abastecen de gran cantidad de material residual de las zonas que presentan altas pendientes.

Segundo, los sectores donde se localizan los depósitos sedimentarios coluviales se encuentran entre los rangos de mayor susceptibilidad. Hauser (1993) y Suárez (2010) concuerdan que los materiales litológicos sedimentarios, especialmente coluviales, y los volcanoclásticos, son los que presentan mayor susceptibilidad a los procesos de remoción de masas en los ambientes andinos. Los resultados del tratamiento cartográfico digital y los trabajos de campo permiten destacar el factor litológico dentro del análisis de movimientos en masa, porque permitió identificar aquellos espacios donde ocurrieron antiguos movimientos de tierra. 
Tabla 1. Matriz cuadrada, peso de factores, peso de clases y razón de inconsistencia, realizados con el Método de Jerarquización Analítica.

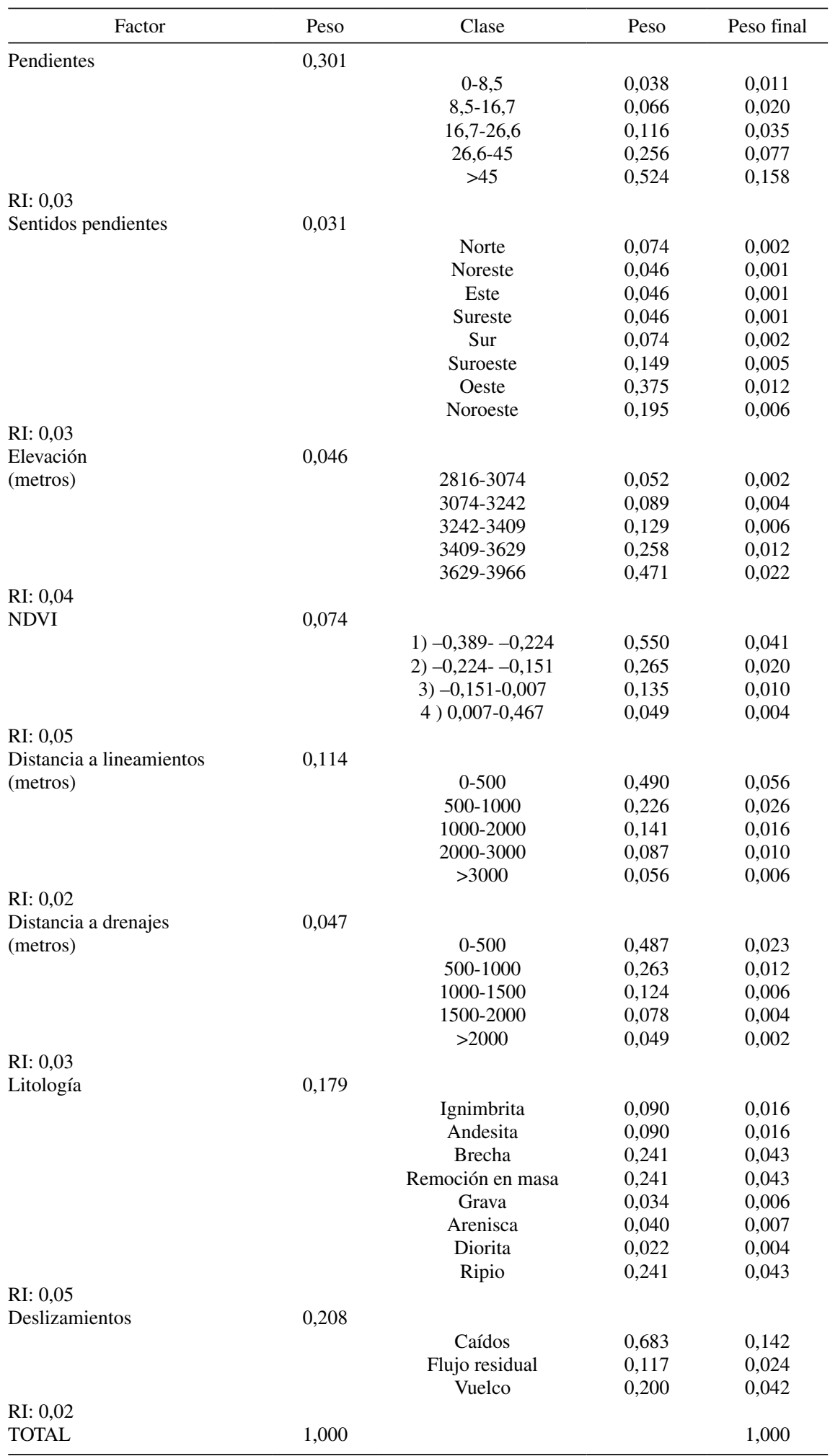




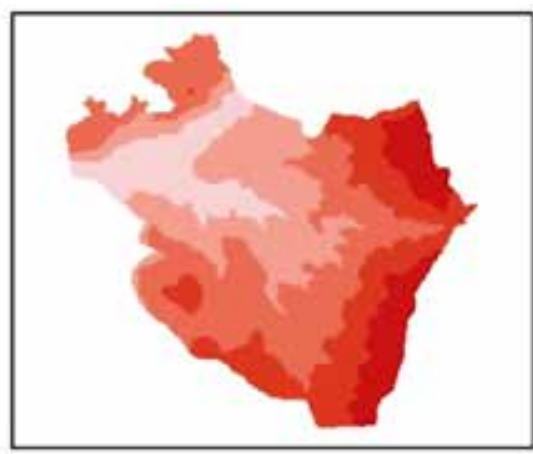

A) Altitud

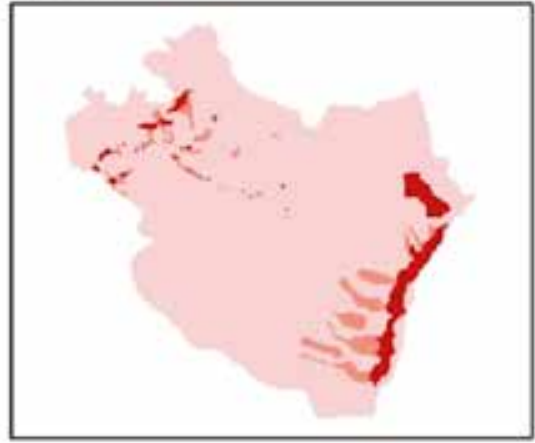

C) Inventario deslizamientos

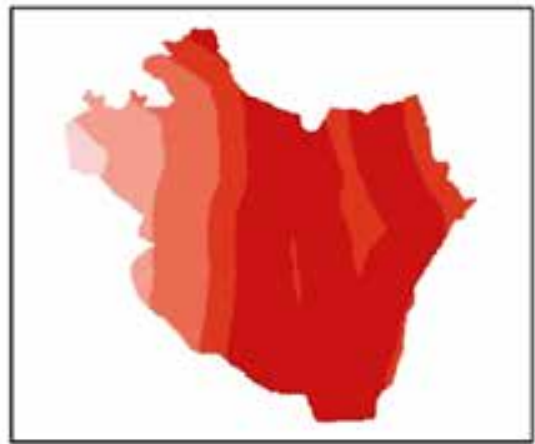

E) Distancia a lineamientos

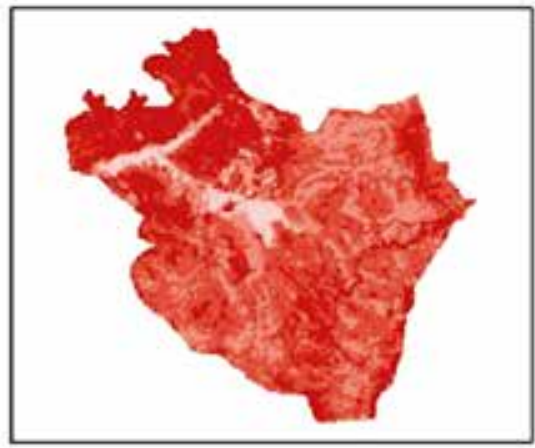

G) NDV!

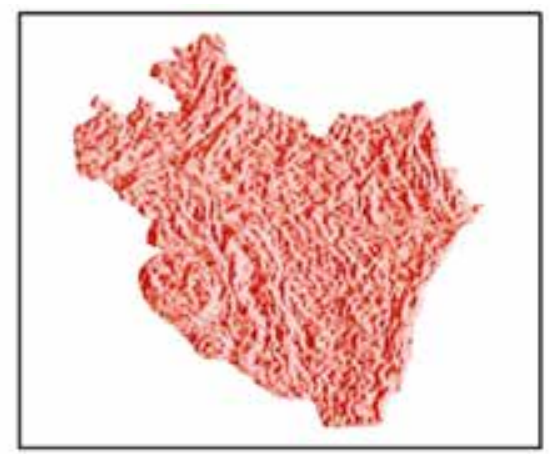

B) Sentido de las pendientes

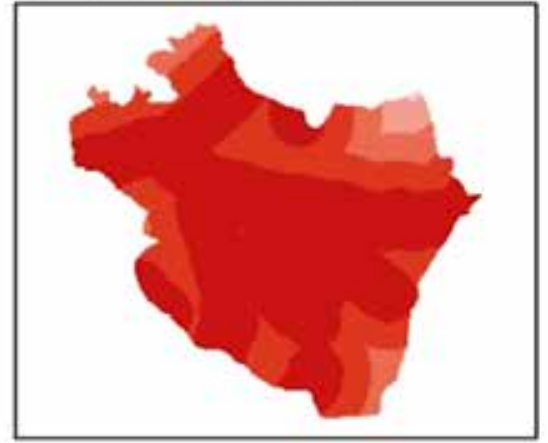

D) Distancia de drenaje

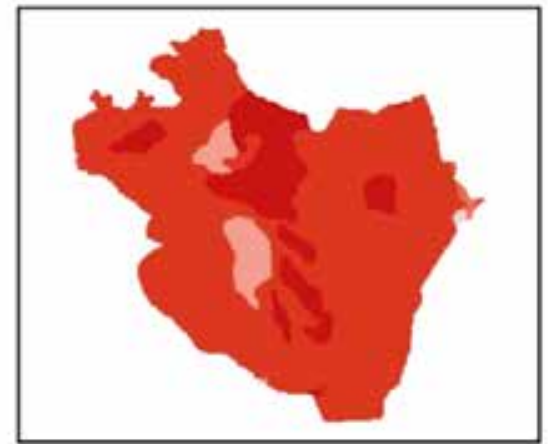

F) Litologia

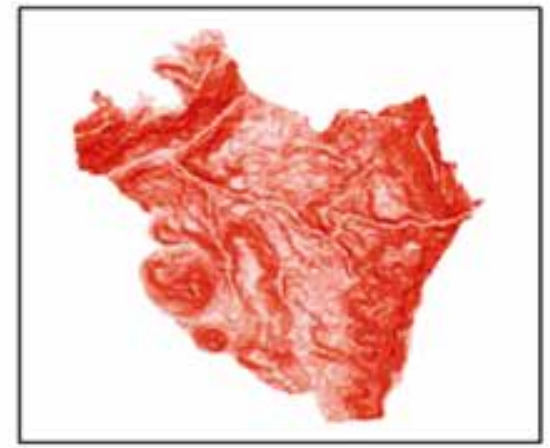

H) Pendientes
LEYENDA

Factores

Valores

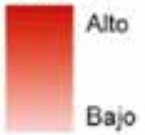

Figura 7. Factores condicionantes, incorporados en el estudio, que favorecen la susceptibilidad de los movimientos en masa. 
Tabla 2. Índice de susceptibilidad a delizamientos y puntos críticos. Elaborado a partir de los resultados de la Tabla 1.

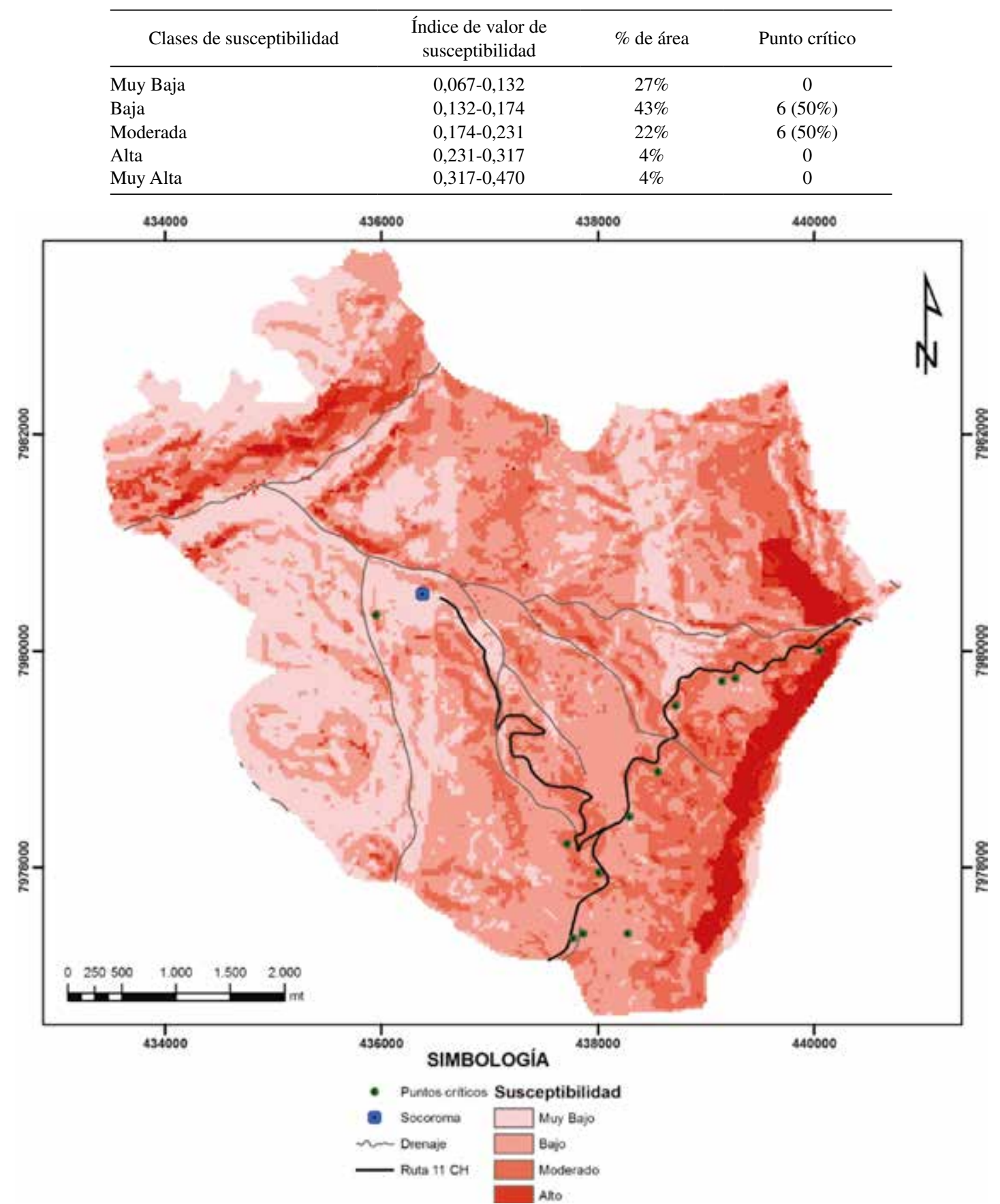

Figura 8. Zonificación: rangos de susceptibilidad, escala 1:50.000.

No obstante, en el área estudiada no parecen ser el principal factor a tener en cuenta; pero sí permite discriminar aquellos depósitos proclives a la inestabilidad, que también están relacionados con la misma estructura de la roca.
Tercero, los lineamientos estructurales posibilitan rangos de susceptibilidad muy altos. Hauser (1993) menciona que las zonas de falla y/o fracturas propician desarrollos geomecánicamente débiles que favorecen a los procesos de remoción 
en masa. Según los resultados obtenidos podemos inferir que estas estructuras geológicas juegan un rol importante dentro del análisis de movimientos en masa, no solamente por el peso otorgado en el análisis multicriterio de este trabajo, sino por su influencia en la constitución geomorfológica del área estudiada, ya que el relieve asociado a estas estructuras presenta accidentes importantes en los que se activan flujos detríticos como los que cortan la ruta internacional 11-CH. El plano de la falla Socoroma se convierte en un elemento clave para comprender la localización y sectorización de rangos de susceptibilidad muy altos, e influye considerablemente en los factores morfométrico y litológico del área estudiada.

\section{Conclusión}

A partir de los resultados obtenidos del análisis multicriterio se concluye que dentro del área estudiada existen sectores que poseen un alto índice de susceptibilidad de movimientos en masa, lo que favorecería la ocurrencia de estos fenómenos en el futuro.

Se puede inferir que aquellos sectores donde la ladera está compuesta por cornisas, poseen la más alta probabilidad de que se produzcan deslizamientos de roca. Como se aprecia en la Figura 8, el sector noroeste y suroeste presentan altos índices de susceptibilidad a movimientos, principalmente en las partes altas de las laderas o taludes, en cuyas bases pueden encontrarse depósitos de derrubios como testigos de la actividad presente.

La localidad de Socoroma, principal punto poblado del área de estudio, se encuentra localizada en un sector de baja susceptibilidad y de relativa estabilidad, rodeada por sistemas de andenerías que favorecen la estabilidad de las pendientes.

En el sector noroeste, la actividad agrícola se encuentra expuesta a los caídos de roca, flujos residuales y avalanchas de detritos de mayores dimensiones - estos últimos denominados por la población local como Lloclla (agua sucia). Las avalanchas de detritos se desencadenan cuando los caudales de agua, en los escurrimiento principales, aumentan significativamente, siendo capaces de movilizar gran cantidad de materiales de distinto tamaño.

El sector sureste, en la parte alta de sus laderas, presenta salientes rocosas que suministran material detrítico al sector donde se presenta el plano de falla Socoroma, y con el paso del tiempo son removilizadas pendiente abajo, y han dado origen a extensos depósitos de coluvión, que son testigos de importantes movimientos en masa ocurridos en el pasado.

Finalmente, la ocurrencia de estos fenómenos provocan la incomunicación de la localidad de Socoroma y del resto del hinterland con el sector costero de la región de Arica y Parinacota, al producirse cortes en la carretera internacional 11-CH, lo que se traduce en aislamientos de la población y pérdidas económicas consiguientes; trayendo consigo además el aislamiento de Bolivia con el puerto de Arica, principal vinculo de salida y entrada de los productos del comercio internacional boliviano.

\section{Agradecimientos}

El presente trabajo se ha materializado gracias a la ayuda y aporte de varias personas. Deseamos agradecer a la comunidad de San Francisco de Socoroma, personas muy amables y cordiales. En especial al presidente de la Junta de Vecinos, el señor Julián Mamani; y a la señora Filomena Emilia Humire, quien accedió amablemente a responder nuestras consultas. También agradecemos la ayuda desinteresada del profesor señor Juan José Sanz Donaire, por hacernos llegar sus observaciones del presente manuscrito, lo que valoramos enormemente. A todos les damos nuestros sinceros agradecimientos.

\section{Referencias Citadas}

\footnotetext{
Álvarez, L.

1987 El mito del Pusiri Collo y la fiesta del Pachallampe: aculturación andino-hispana en el poblado de Socoroma. Diálogo Andino 6: 80-90.

Ram Avtar, C., Singh, K., Gulab Singh, Verma, R. L., Mukherjee, S. y Sawada, H.

2013 Landslide susceptibility zonation study using remote sensing and GIS technology in the Ken-Betwa River Link area, India. Bulletin of Engineering Geology and the Environment 70 (4): 595-606.
}

De Pedraza, J., Carrasco, R., Díez, A., Martín, J., Martín, A., y Sanz, M. A.

1996 Geomorfología: principios, métodos y aplicaciones. Editorial Rueda, Madrid.

Díaz, E., Sáenz de Olazagoitia, A., Ormaetxea, O., Ibisate, A. 2012 Analisis de factores de desestabilización de laderas en dos cuencas del ámbito atlántico: Sollube-Mape (Bizkaia) y Ramaio (Alava). Geomorfología y Cuaternario 26 (1-2): 171-190. 
Fuenzalida, $\mathrm{H}$.

1965 Clima. CORFO (1965) Geografía Económica de Chile. Texto refundido, pp. 99-152. Corporación de Fomento de la Producción, Santiago de Chile.

García, M., Gardeweg, M., Clavero, J. y Hérail, G. 2004 Hoja Arica, Región de Tarapacá. Servicio Nacional de Geología y Minería, Carta Geológica de Chile, Serie Geología Básica, 84, 1 mapa escala 1:250.000. SERNAGEOMIN, Santiago de Chile.

Gil, V., Campo, A.

2012 Geomorfología y procesos de vertiente. Cuenca alta del río Sauce Grande (Buenos Aires, Argentina). Cuaternario y Geomorfología 26 (1-2): 133-150.

Golubev, G.

1969 Avalanchas y corrientes de barro en Chile. Revista Informaciones Geográficas 17: 31-74.

Gutiérrez, M.

1998 Geomorfología y cambio climático en zonas áridas. Academia de Ciencias Exactas, Físicas, Químicas y Naturales de Zaragoza. Sociedad Cooperativa de Artes Gráficas, Zaragoza.

Haflidason, H., Lien, R., Sejrup, H., Forsberg, C., Bryn, P. 2005 The dating and morphometry of the Storegga Slide. Marine and Petroleum Geology 22: 123-136.

Hauser, A.

1985 Flujos de barro en la zona preandina de la Región Metropolitana: características, causas, efectos, riesgos y medidas preventivas. Revista Geológica de Chile 24: 75-92.

Hauser, A.

1985 Flujos aluvionales de 1870 y 1896 ocurridos en la ladera norte del volcán Yates, X Región: su implicancia en la evaluación de riesgos naturales. Revista Geológica de Chile 25-26: 125-133.

Hauser, A.

1993 Remociones en Masa en Chile. Servicio Nacional de Geología y Minería, Boletín 45, Santiago de Chile.
Hauser, A.

1997 Los aluviones del 18 de junio de 1991 en Antofagasta: un análisis crítico, a 5 años del desastre. Servicio Nacional de Geología y Minería, Boletín 49, Santiago de Chile.

Hervás de Diego, J. y Barredo Cano, J. I.

2001 Evaluación de la Susceptibilidad de Deslizamientos mediante el uso conjunto de SIG, teledetección y métodos de evaluación multicriterio. Aplicación al barranco de Tirajana (Gran Canaria). V Simposio Nacional sobre Taludes y Laderas Inestables, Madrid, 27-30: 305-316.

Intarawichian, N. y Dasananda, S.

2010 Analytical Hierarchy Process for Landslide Susceptibility Mapping in lower Mae Chaem Watershed, Northern Thailand. Suranaree Journal of Science and Technology 17 (3): 277-292.

Kawabata, D. y Bandibas, J.

2009 Landslide susceptibility maping using geological data, a DEM from Aster images and an Artificial Neural Network (ANN)'. Revista Geomorphology 113: 97-109.

Montecinos, F.

1962 Grietas y deslizamientos de tierra en la región de Socoroma, departamento de Arica. Instituto Investigaciones Geológicas, Santiago, Chile.

Polanski, J.

1966 Flujos rápidos de escombros rocosos en zonas áridas y volcánicas. EUDEBA, Buenos Aires Argentina.

Royán, M., Vilaplana, J.

2012 Distribución espacio-temporal de los desprendimientos de rocas en la montaña de Montserrat. Cuaternario y Geomorfología 26 (1-2): 151-170.

Saaty, T. H.

1980 The Analytic Hierarchy Process: Planning, Priority Setting. McGraw-Hill, New York.

SERNAGEOMIN

2003. Mapa Geológico de Chile. Versión digital. Servicio Nacional de Geología y Minería, Publicación Geológica Digital, 4 (CD-ROM, versión 1.0, 2003). Santiago.

Suárez, J.

2010 Deslizamientos: Análisis geotécnico. 1. Universidad de Santander, Colombia. 
